\title{
FORUM
}

\section{Hovercraft and the Collision Regulations}

\author{
from Commander P. C. H. Clissold, R.N.R.
}

IN the discussion which followed the papers on Hovercraft Navigation, Captain M. J. Edwards raised an important point. 'If the hovercraft is the stand-on vessel, it may be impossible for a large slow-moving vessel to take avoiding action in the short period between sighting and collision.' This implied that both were following the International Collision Regulations, and these cannot be altered (except by local authorities in 'inland waters') without international agreement and the attendant delay.

The question raised was 'what steps are being taken to resolve the general collision problem on the high seas?'

The steps taken may be slow, but they must be started. A hovercraft is undoubtedly a 'vessel' within the meaning of the Rules, in that it is 'used, or capable of being used, as a means of transportation on water.' (Rule I (c) (i)), but a seaplane is specially excepted from this classification of 'vessel', though it too is capable of being used as a means of transportation on water. From LieutenantCommander P. M. Lamb's reply to Captain Edward's question it seems that the hovercraft captain would prefer his vessel to be regarded as a 'seaplane' which 'shall, in general, keep well clear of all vessels and avoid impeding their navigation. In cases, however, where risk of collision exists, she shall comply with the Rules (Rule 2o(b)).' If this classification was generally accepted as satisfactory it would mean the least possible interference in the Rules: simply the insertion of the one word 'hovercraft' in Rule I(c)(ii) which would then read:

"The word "seaplane" includes a hovercraft, a flying boat and any other aircraft designed to manœuvre on the water.'

It is clear that in cases where one vessel is very fast and the other vessel much slower avoiding action in almost any state of visibility except the clearest, must be taken by the faster because effective action cannot be taken by the slower. We shall probably soon find hydrofoil craft, as well as hovercraft, operating outside the 'inland waters' defined in Rule 30 . Conventional high-speed launches already exist and probably act in the same way as 'a seaplane on the water' in general keeping 'well clear of all vessels,' but when risk of collision exists complying with the Rules.

We thus seem likely to be confronted with a class of vessels, not envisaged in the Rules, of an opposite type to those vessels who are 'unable to manœuvre as required by these Rules' and who indicate this fact by displaying special signals so that ordinary vessels can avoid them.

Perhaps we should drop all reference to seaplanes (except for their extra anchor lights) and substitute a new Rule for Very Fast Vessels. This might read something like Rule 20 (b):

'(a). Very fast vessels, such as hovercraft, hydrofoil craft and seaplanes on the water, shall, in general, keep well clear of all vessels and avoid impeding their 
navigation. In cases, however, when risk of collision exists, they shall comply with these Rules.

(b). By night they shall carry, in addition to the lights prescribed for other vessels of their class and tonnage,-' some specified light such as the flashing amber light referred to by Lieutenant Commander J. H. Hardwick.

\title{
A Theoretical Note on System Malfunctioning
}

\author{
from J. B. Parker
}

THE problem of estimating equipment serviceability when the apparatus consists of a large number of individual components (possibly with standby features) is likely to be of increased interest now that navigational thought is being concentrated quite as much on the design of instruments the navigator uses as on the problems of finding position at sea or in the air. The resulting exercise in combining probabilities of component failure is a fairly straightforward one but in view of the accelerating interest in the matter (see, for example, Cluley's article in this Journal, 15, 387) a brief systematic treatment might be of interest to workers in these fields.

The basic concept is the failure rate of a component. It is in the specification and interpretation of this quantity that a possible pitfall lies. A rate implies time and one must guard against interpreting failure rates as if they were probabilities. A simple example will suffice. If a failure rate of say $10^{-3}$ per hour is specified, does it mean that after 500 hours the equipment has a 50 per cent survival chance? Is it bound to be inoperative after r ooo hours?

The answer is no and to interpret the failure rate it is of interest to consider the analogy of a radioactive substance. This consists of a large number of atoms each of which decays with known 'half life'. When the 'half life' is spent, half the material will have decayed but it is not true that after two 'half-lives' all the material will have been transformed.

Let us imagine an equipment with failure rate $\alpha$ (alternatively, a radioactive substance with decay rate $\alpha$ ). At time $t$ we imagine a large number of identical equipments (or atoms), $N(t)$. Then, after a short duration of time $\Delta t$, a proportion $\alpha \Delta t$ of the product will have become unserviceable. Thus

$$
N(t+\Delta t)=N(t)\{1-\alpha \Delta t\}
$$

As $\Delta t$ shrinks to zero this equation leads to the differential equation

with solution

$$
\frac{d N}{d t}^{(t)}=-\alpha N(t)
$$

$$
N(t)=N(o) \exp -\alpha t
$$

Thus however long the duration of time, there will always be some chance, $N(t) / N(o)=\exp -\alpha t$, of the equipment continuing to function correctly.

The conclusion is that in mathematical problems where failure rates are of 